\title{
COMPLICAÇÕES RESPIRATÓRIAS NO PÓS-OPERATÓRIO
}

\author{
POSTOPERATIVE RESPIRATORY COMPLICATIONS
}

Alfredo José Rodrigues, Paulo R. Barbosa Évora, Walter Villela de A. Vicente

Docentes da Divisão de Cirurgia Torácica e Cardiovascular do Departamento de Cirurgia e Anatomia da FMRP-USP

CorRespondência: Alfredo José Rodrigues.Divisão de Cirurgia Torácica e Cardiovascular do Departamento de Cirurgia e Anatomia da FMRP-USP. Av. Bandeirantes, 3900, 14049-900 - Ribeirão Preto / SP. (email: alfredo@ fmrp.usp.br)

Rodrigues AJ, Évora PRB, Vicente WVA. Complicações respiratórias no pós-operatório. Medicina (Ribeirão Preto) 2008; 41 (4): 469-76.

RESUMO: As complicações relacionadas ao sistema respiratórias não são incomuns no pósoperatório de operações de maior porte e aumentam a morbidade e mortalidade. Entre as mais freqüentes estão a atelectasia, a pneumonia, o tromboembolismo pulmonar e a falência respiratória. No presente artigo são revisados resumidamente os aspectos gerais da fisiopatologia, a apresentação clínica, o diagnóstico e o manuseio clínico dessas entidades.

Descritores: Complicações Pós-operatórias. Tromboembolismo. Atelectasia. Pneumonia. Insuficiência Respiratória.

\section{1- INTRODUÇÃO}

As complicações relacionadas ao sistema respiratórias não são incomuns no pós-operatório e aumentam a morbidade e a mortalidade. Entre as mais frequientes estão a atelectasia, a pneumonia, o tromboembolismo pulmonar e a falência respiratória. Em uma recente revisão para o "American College of Physici-ans" vários fatores de risco para complicações pulmonares com níveis de evidência considerados ao menos satisfatórios foram identificados. ${ }^{1,2} \mathrm{~A}$ idade avançada (o risco aumenta progressivamente, sendo que a partir de 60 anos a razão de risco (RR) é de 2,0 e acima de 70 anos de 3,0), classificação de risco anestésico pela "American Society of Anesthesiologists" $\geq$ 2 (RR 2,5 a 4,8), a presença de insuficiência cardíaca (RR 2,9), a dependência total ou parcial de ajuda para o desempenho de atividades diárias (RR 2,5) e a Doença Pulmonar Obstrutiva Crônica (RR 1,8) são todos considerados fatores para os quais os níveis de evidência são considerados bons. Há ainda evidências menos conclusivas que sugerem que o rebaixamento do nível de consciência, anormalidades no exame físi- co dos pulmões (estertores, roncos, sibilos e expiração prolongada), o tabagismo ativo, o alcoolismo e perda de peso $>10 \%$ nos últimos 6 meses também podem expor os pacientes a maior risco de complicações respiratórias. Ademais, alguns procedimentos cirúrgicos, e fatores a ele relacionados, representam maior risco para os pacientes. Assim, as operações para correção de aneurisma da aorta, as operações torácicas, as operações abdominais altas, vasculares, envolvendo a cabeça ou pescoço e as neurocirurgias são todas operações com risco considerável $(\mathrm{RR}>2)$. Ademais, a anestesia geral, operações realizadas em caráter de emergência, tempo de cirurgia prolongado $(>2,5 \mathrm{~h}) \mathrm{e}$ a necessidade de transfusão no perioperatório são foram identificados como fatores de risco adicionais.

De uma forma geral os principais fatores envolvidos na fisiopatologia das complicações respiratórias no pós-operatório são: 3,4

1) Dor pós-operatória: principalmente por limitar a ventilação e a tosse.

2) A anestesia geral e os analgésicos: A manipulação das vias aéreas pode propiciar contaminação e as drogas anestésicas, assim como a mani- 
pulação das vias aéreas superiores, podem interferir com a produção de muco e movimentação ciliar (mecanismos de defesa). Ademais, os anestésicos, os analgésicos e os relaxantes musculares, sobretudos os de ação mais prolongada, interferem com a consciência e a força muscular, respectivamente, diminuindo a ventilação e o reflexo da tosse. A ventilação com alta fração inspirada de oxigênio durante o período perioperatório propicia maior reabsorção do ar alveolar e pode ser responsável por lesão pulmonar. ${ }^{5}$

3) As incisões, sobretudo toracotomias e abdominais alta, além da dor, rompem a integridade muscular, resultando em disfunção da musculatura respiratória.

4) As operações em órgãos abdominais resultam em diminuição da função do diafragma por interferência com os impulsos nervosos para a musculatura respiratória, sobretudo o diafragma. ${ }^{4}$

5) No pós-operatório ocorre demanda aumentada de oxigênio, o que pode precipitar a insuficiência respiratória em pacientes com reserva pulmonar limitada por disfunção pré-existente, decorrente de doença pulmonar, ou adquirida no perioperatório (atelectasia, por exemplo), ou mesmo em decorrência das alterações inerentes ao envelhecimento.

6) A disfunção cardíaca pré-existente, ou devido a eventos perioperatórios tais como infarto e/ou depressão por drogas, ou mesmo como efeito da resposta inflamatória sistêmica perioperatória, podem precipitar congestão pulmonar e baixo débito cardíaco.

7) A resposta inflamatória sistêmica, basicamente a liberação de diversos fatores inflamatórios, como citocininas e leucotrienos, com a ativação de leucócitos e plaquetas, em resposta ao trauma cirúrgico, podem resultar em lesão orgânica, pulmonar inclusive.$^{6,7}$

\section{2- ATELECTASIA}

A atelectasia nada mais é do que o colapso de porção variável do parênquima pulmonar. É a complicação pulmonar mais comum no pós-operatório e a causa mais comum de febre nas primeiras 48 horas de pós-operatório. A sua fisiopatologia esta intimamente relacionada à deficiência da produção de surfactante, reabsorção do ar alveolar e hipoventilação, pelas causas já expostas. Do ponto de vista fisiopatológico re- sulta basicamente em alvéolos perfundidos, mas não ventilados, o chamado "shunt intrapulmonar", podendo acarretar desaturação do sangue arterial pela mistura desse sangue que passa por esses alvéolos não ventilados e, portanto, sem a ocorrência da hematose. ${ }^{4}$

\section{1- Quadro Clínico}

O sintoma mais comum é a febre, usualmente baixa. Pode ocorrer insuficiência respiratória de grau variável na dependência da reserva funcional prévia e do grau de colapso do parênquima pulmonar. Assim as manifestações respiratórias podem variar de sinais de disfunção respiratória leve até franca insuficiência respiratória com evidente dispnéia, diminuição da saturação de oxigênio arterial e da pressão parcial do oxigênio arterial $\left(\mathrm{PaO}_{2}\right)$, taquicardia, taquipnéia e cianose. Ademais, a atelectasia pode se complicar com infecção pulmonar.

\section{2- Diagnóstico Radiológico}

Habitualmente a atelectasia envolvendo porção de parênquima correspondente ao menos a um segmento pulmonar é passível de identificação à radiografia simples de tórax (Figura 1). Nos casos mais evidentes, geralmente envolvendo vários seguimentos ou um ou mais lobos, pode-se verificar na radiografia simples de tórax a presença de condensação (opacidade) de grau variável, usualmente sem broncograma aéreo, acompanhada de sinais de perda de volume no hemitórax acometido (elevação de cúpula frênica, aproximação de cissuras, desvio ipsilateral da traquéia e/ ou coração e grandes vasos) e por vezes há expansão compensatória nítida do pulmão contralateral, sobretudo nos casos crônicos. Pequenas porções de atelectasia do parênquima podem se mostrar à radiografia como faixas horizontalizadas, ou obliquas, em direção ao hilo pulmonar, ou condensações intralobares sem perda volumétrica nítida. A tomografia computadorizada de tórax é o melhor exame para a detecção das atelectasias, pois sua sensibilidade é superior a da radiografia simples, permitindo a visualização de atelectasia de proporções bem menores de parênquima habitualmente não identificáveis à radiografia. Todavia, há de se considerar que a tomografia computadorizada é pouco utilizada na prática clínica diária para o diagnóstico de atelectasia em nosso meio, uma vez que a relevância clínica de uma atelectasia não identificável à radiografia simples de tórax é questionável. 

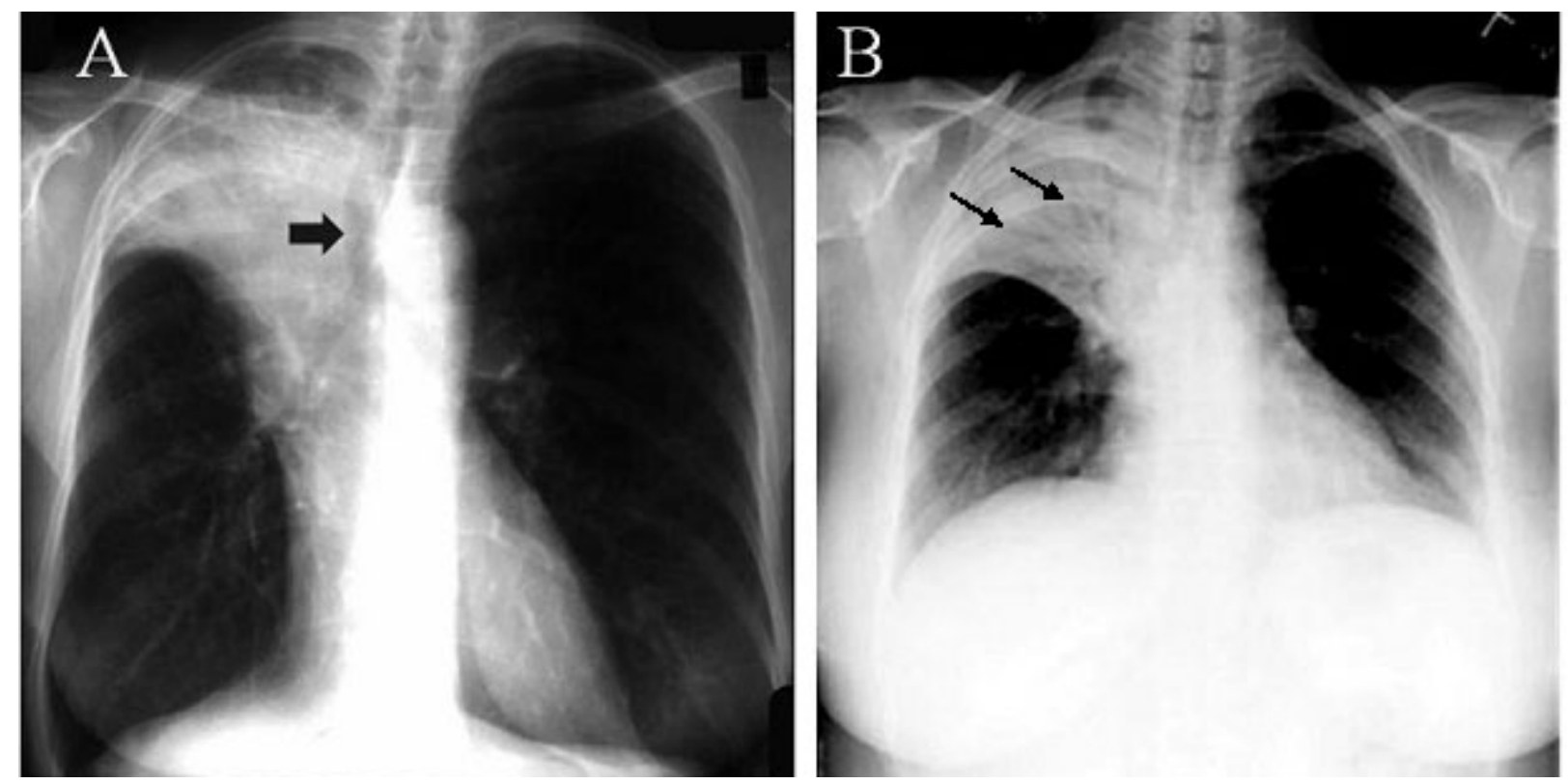

Figura 1: A radiografia "A" mostra atelectasia de lobo superior direito. Observa-se, além da opacidade acometendo a porção apical do hemitórax direito, o desvio da traquéia para a direita (seta negra) e a redução volumétrica desse hemotórax, em comparação ao contralateral. Na radiografia "B", a direita, os achados radiológicos são semelhantes, mas trata-se de uma penumonia lobar superior direita. Não há perda de volume em comparação ao outro hemitórax, nem desvio da traquéia. Ademais, nota-se a presença de broncogramas aéreos (setas finas) que indicam brônquios pérvios, com ar no interior.

\section{3- Estratégia para prevenção e reversão: ${ }^{1}$}

1) Menor dano tecidual: Cirurgias minimamente invasivas (laparoscopia p.e.);

2) Analgesia adequada;

3) Mobilização precoce no pós-operatório, propiciando melhora da ventilação;

4) Hidratação adequada, propiciando fluidificação das secreções;

5) Exercícios respiratórios:

a) Estimular o paciente a tossir e expectorar;

b) Estimular a realização de exercícios respiratórios com inspirações profundas, espontâneas ou com uso de aparatos apropriados. Todavia, não há evidências sólidas de que o uso de aparatos específicos para incentivar as inspirações espontâneas profundas, ou outros para ampliar o volume inspirado, seja superior às inspirações espontâneas não aparelhadas.

Para o tratamento da atelectasia estabelecida aplicam-se as mesmas medidas utilizadas para a profilaxia, podendo-se lançar mão de aparelhos que geram fluxo adicional durante a inspiração ("RPPI": pressão positiva intermitente nas vias aéreas) ou que aplicam pressão positiva constante durante a todas as fases da respiração ("CPAP": pressão positiva contínua nas vias aéreas) ou diferenciadamente nas duas fases da respiração ("BIPAP": pressão positiva com dois níveis nas vias aéreas). Todavia, como para a profilaxia, a fisioterapia respiratória aparelhada carece de evidências que suportem sua superioridade em comparação à não aparelhada.

\section{3- PNEUMONIA}

Os dois principais tipos de pneumonia que podem ocorrer no pós-operatório são a pneumonia bacteriana hospitalar e a pneumonite aspirativa. ${ }^{3,8,9}$

\section{1- Pneumonite aspirativa}

É decorrente da aspiração do conteúdo gastrointestinal e oral no período pós-operatório. Inicialmente causa irritação das vias aéreas devido a ação direta do suco gástrico e entérico e pode ocorrer a contaminação das vias aéreas e pulmões por bactérias presentes nestas secreções (geralmente anaeróbios e gram-negativos). 
A inflamação local inicial pode evoluir em poucas horas com intensa inflamação pulmonar com atelectasia, infiltrado inflamatório e edema ou mesmo hemorragias do parênquima e disfunção respiratória progressiva de grau variado. ${ }^{9}$ Inicialmente o diagnóstico é presuntivo e o paciente pode estar assintomático ou apresentar dispnéia com estertores, roncos ou sibilos e febre baixa. A radiografia de tórax pode mostrar, usualmente várias horas após o evento inicial, infiltrado alveolar bilateral, mais pronunciado nas regiões peri-hilares e/ou basais, ou nos seguimentos apical dos lobos superiores e apicais dos lobos inferiores. O quadro pode evoluir com edema pulmonar não cardiogênico e insuficiência respiratória com hipoxemia e mesmo hipercapnia.

O tratamento baseia-se na terapia de suporte com hidratação adequada, medidas para evitar novos episódios de aspiração, suplementação de oxigênio ou assistência ventilatória mecânica, invasiva ou não-invasiva, conforme a necessidade. A pneumonia bacteriana associada não é rara, portanto se o quadro clínico se deteriora após as primeiras $24 \mathrm{~h}$ a utilização de antibióticos visando cobrir anaeróbios e gran-negativos deve ser considerada. O emprego de corticosteróides é controverso e não há evidências suficientes que justifiquem sua utilização rotineira. ${ }^{9}$

\section{2- Pneumonia Bacteriana nosocomial pós-ope- ratória}

Pneumonia é a terceira infecção pós-operatória mais comum, após a infecção urinária e de ferida operatórias, e esta associada a alta mortalidade. Os principais fatores envolvidos na patogênese da pneumonia no perioperatório são a colonização do paciente com a flora hospitalar, as alterações do sistema imunológico provocadas pela patologia de base e/ou trauma cirúrgico, ${ }^{6}$ e as diversas circunstâncias que propiciam a aspiração, nem sempre macroscópica, de conteúdo oral e/ou entérico (anestesia geral, emprego de canulas orotraqueais, sedação, sonda nasogástrica, distensão do trato digestivo e diminuição da efetividade da tosse). As bactérias gran-negativas estão envolvidas em 55\%-85\% dos casos e as gran-positivas em 20-30\%. Todavia em 40 a 60\% dos casos a infecção é polimicrobiana. Os principais agentes isolados são: Streptococcus pneumoniae, Staphylococcus aureus, Klebsiella pneumoniae, E coli, Haemophilus influenzae, Pseudomonas aeruginosa, Enterobacter cloacae, Serratia marcescens. Os fatores de risco, além daqueles apontados para as complicações respiratórias em geral, são a presença de disfunção renal, acidente vascular cerebral prévio e uso de corticesteróides.

\section{3- Diagnóstico}

O diagnóstico é difícil, uma vez que a presença de febre e alterações pulmonares à radiografia não significam necessariamente que haja infecção, pois contusão pulmonar, atelectasia e a Síndrome da Angustia Respiratória Aguda (SARA) podem promover febre com alterações radiológicas semelhantes.

Sinais clínicos e achados sugestivos de pneumonia no pós-operatório são: ${ }^{8}$

1) Infiltrado pulmonar novo ou progressão de um préexistente à radiografia de tórax,

2) Febre

3) Leucocitose $>10.000 / \mathrm{mm}^{3}$,

4) Escarro purulento

5) Piora da função respiratória

6) Obtenção do patógeno a partir de hemoculturas, ou do lavado brônquico, ou do aspirado transtraqueal, ou ainda de biopsia pulmonar.

O tratamento deste tipo de infecção é complexo pelo fato de se tratar de pacientes com comorbidades e com flora não usual. Assim, a escolha do antibiótico deve se basear nas culturas obtidas da diretamente das vias respiratórias mediante broncofibroscopia (lavado ou escovado, preferencialmente quantitativo), ou punção traqueal ou da hemocultura. Até que se tenham estes resultados, a escolha do antibiótico deve se basear no tipo de germe comumente associado a infecções desse tipo no hospital em questão ou na unidade específica daquele hospital (UTI, por exemplo), na condição do paciente e na possibilidade do envolvimento de certas tipos de bactérias (Staphylococcus aureus "resistentes", Acinetobacter baumannii e Pseudomonas aeruginosa). Assim a comissão de infecção hospitalar sempre deve ser acionada para ajudar na escolha do antibiótico mais apropriado. Para o tratamento empírico (> $80 \%$ atividade) os antibióticos de eleição são: vancomincina, carbapenems (imipenem, meropenem), piperacilina, cefepime, ceftazidime, ciprofloxacin, e aminoglicosídeos.

\section{4- INSUFICIÊNCIA RESPIRATÓRIA NO PÓS-OPERATÓRIO}

Uma ampla variedade de patologias pode resultar em insuficiência respiratória no pós-operatório. Basicamente a insuficiência respiratória pode ser dividida em 3 tipos. 
1) Tipo I ou hipoxêmica ou por "falência de oxigenação": Caracterizada por baixa $\mathrm{PaO}_{2}$ com $\mathrm{PaCO}_{2}$ normal ou baixa (hiperventilação) e geralmente associada a distúrbios da relação ventilação/perfusão. São desse tipo as provocadas pela Síndrome da Angustia Respiratória Aguda (SARA) ou Lesão Pulmonar Aguda, Edema Pulmonar Cardiogênico, Pneumonia, Embolia Pulmonar, Atelectasia, Asma e DPOC.

2) Tipo II ou hipercápnica ou "falência de ventilação": Caracterizada por baixa $\mathrm{PaO}_{2}$ com $\mathrm{PaCO}_{2}$ elevada (hipoventilação). As principais causas no pós-operatório são o rebaixamento do nível de consciência e/ou bloqueio neuro-muscular.

3) Tipo III ou Mista: Como a tipo II também é caracterizada por baixa $\mathrm{PaO}_{2}$ e $\mathrm{PaCO}_{2}$ elevada (hipoventilação ou diminuição da difusão de $\mathrm{CO}_{2}$ nas fases adiantadas de dano pulmonar) e as principais causas são a Asma, DPOC e SARA.

No pós-operatório o edema pulmonar cardiogênico ${ }^{10}$ é decorrente da elevação da pressão capilar pulmonar como conseqüência da piora da disfunção ventricular esquerda e/ou valvar aórtica e/ou mitral pré-existentes, ou cuja função era limítrofe, no qual o efeito negativo de drogas anestésicas, a reação inflamatória sistêmica associada ao trauma cirúrgico, que pode produzir fatores depressores do miocárdio, e não raramente hipervolemia iatrogênica, podem estar associados.

Em contraste com o edema pulmonar cardiogênico, no qual o aumento da pressão hidrostática no capilar pulmonar é o fator primordial, na LPA e a SARA a disfunção e o edema estão relacionados ao envolvimento dos pulmões em um complexo processo inflamatório que resulta em infiltração de células inflamatórias no interstício e alvéolos, no aumento da permeabilidade capilar com edema intersticial e alveolar, ou mesmo hemorragia intra-alveolar, disfunção de pneumócitos e, mais tardiamente, fibrose intersticial. ${ }^{3,6,7} \mathrm{O}$ envolvimento pulmonar na LPA é menos severo e de duração mais curta. A Tabela I expõe os critérios para a diferenciação dessas três entidades.

O tratamento de cada uma destas entidades foge ao escopo desta revisão, mas em geral o tratamento do edema pulmonar cardiogênico envolve o uso de diuréticos, vasodilatadores e drogas inotrópicas positivas com suplementação de $\mathrm{O}_{2}$ e na SARA e LPA geralmente a utilização de ventilação mecânica invasiva ou não-invasiva é necessária.

\section{5- TROMBOEMBOLISMO PULMONAR}

O tromboembolismo pulmonar caracteriza-se pela oclusão do sistema arterial pulmonar por embolo de origem trombótica. Mais de $90 \%$ dos êmbolos se originam nas veias profundas dos membros inferiores ou pelve. ${ }^{11,12}$

$\mathrm{Na}$ fisiopatologia da trombose venosa os principais fatores envolvidos são: ${ }^{11}$

1) Estase venosa prolongada devido imobilização e/ ou a disfunção venosa nos membros inferiores

2) Lesão ou Ativação Endotelial:

Há de se considerar que embora as veias da pelve e membros inferiores possam ser diretamente lesadas por trauma cirúrgico, por posicionamento inadequado dos pacientes, por cateteres venosos ou mesmo devido a pressão venosa elevada da insuficiência venosa crônica, a resposta inflamatória local e sistêmica que se segue a um trauma, inclusive o trauma cirúrgico, pode resultar na "ativação endotelial", situação na qual o endotélio diminui a produção de substância vasodilatadoras, aumenta

Tabela I: Diferenciação de edema pulmonar cardiogênico, LPA e SARA.

\begin{tabular}{lll}
\hline Edema Cardiogênico & LPA & SARA \\
\hline $\mathrm{PCP} \geq 18 \mathrm{mmHg}$ & $\mathrm{PCP}<18 \mathrm{mmHg}$ & $\mathrm{PCP}<18 \mathrm{mmHg}$ \\
Infiltrado Peri-hilar ou & $\mathrm{PaO}_{2} / \mathrm{FiO}_{2}<300 \mathrm{mmHg}$ & $\mathrm{PaO}_{2} / \mathrm{FiO}_{2}<200 \mathrm{mmHg}$ \\
em "asa de borboleta" & Infiltrado pulmonar bilateral ao RX & Infiltrado pulmonar bilateral ao RX \\
Início agudo & Início agudo & Início agudo \\
História de sobrecarga hídrica & Pode ou não estar associado & Pode ou não estar associado \\
e/ou cardiopatia & à disfunção Cardíaca & à disfunção Cardíaca \\
PCP: pressão capilar pulmonar & &
\end{tabular}


a expressão de proteínas de adesão em sua superfície e de fatores que estimulam a coagulação, a adesão plaquetária e a vasoconstrição (tromboxano e endotelinas, p.e.). Tal ativação costuma ser parte do processo de resposta sistêmica ao trauma operatório. $^{6}$

3) Estados de hipercoagulabilidade

Não apenas a resposta sistêmica ao trauma operatório pode resultar em hipercoagulabilidade e propensão a tromboses, ${ }^{6}$ mas a presença de comorbidades e a patologia de base, tais como neoplasias malignas, uso de estrógenos, tabagismo e distúrbios hematológicos (déficit de Fator $\mathrm{V}$, proteína $\mathrm{C}$, proteína $\mathrm{S}$, antitrombina III, distúrbios do plasminogênio, policitemia, leucocitose, trombocitose, Sindrome Anti-fosfolipídio, p.e) podem estar associadas a propensão para a formação de trombos.

A Tabela II mostra os fatores de risco para o tromboembolismo pulmonar.

\section{1- Diagnóstico (Figura 2)}

Geralmente o tromboembolismo pulmonar se apresenta com dispnéia, dor torácica aguda de intensidade variável e tosse. Habitualmente o quadro clínico se inicia subitamente e a taquipnéia é freqüiente. A hemoptise, embora não freqüente, pode ocorrer, e nos casos mais graves pode haver cianose e franca insuficiência respiratória. Se a embolia é maciça (envolvendo a maior parte da vasculatura pulmonar distal ou o tronco e ramos principais da artéria pulmonar) ocorre súbita elevação da pressão arterial pulmonar e diminuição do enchimento ventricular esquerdo, resultando em hipotensão, ou mesmo choque circulatório cardiogênico, com sinais de disfunção ventricular direita (estase jugula, PVC elevada, congestão hepática). Assim, a presença de trombose venosa, taquicardia com freqüência $>100$ bpm, imobilização ou cirurgia recente, história prévia de tromboembolismo ou trombose venosa, a presença de hemoptise e a presença de neoplasia maligna são altamente sugestivos de tromboembolismo pulmonar.

No eletrocardiograma podem ocorrer alterações inespecíficas do segmento ST e inversão de onda T. Nos casos graves o ECG pode apresentar o padrão S1 Q3 T3 ou desvio para a direita do eixo QRS ou padrão de bloqueio de ramo direito.

A radiografia de tórax é pouco sensível e inespecífica, mas alguns sinais podem ser sugestivos. A rarefação da vasculatura pulmonar em um lobo ou
Tabela II: Fatores de risco para o tromboembolismo pulmonar

- Idade > 60 anos

- Operação extensa*

- Obesidade

- Trombose venosa prévia

- Imobilização prolongada pré e/ou pós-operatória

- Operação ortopédica de grande porte Quadril

Joelho

- Fratura pelve, fêmur ou tíbia

- Neoplasia maligna

- Sepse pós-operatória

- Doença maior associada

Insuficiência cardíaca

Doença Inflamatória Intestino Grosso

Sepse

Infarto do Miocárdio

* Risco de trombose aumenta com a idade, presença de varizes, obesidade e duração da operação.

seguimento pulmonar pode ser observada, ou mesmo uma condensação pulmonar no caso de infarto pulmonar. O achado radiológico de condensação pulmonar periférica em cunha com o ápice voltado para o hilo ("Hampton's hump"), freqüentemente citado, mas incomum e inespecífico, pode estar presente quando há infarto pulmonar.

A dosagem de dímeros-D no plasma tem papel importante na triagem inicial dos pacientes suspeitos de apresentarem tromboembolismo pulmonar. Devido sua alta sensibilidade um resultado negativo para dímeros-D praticamente descarta o diagnóstico de tromboembolismo. Todavia, esse exame não é específico para o tromboembolismo, podendo fornecer resultados positivos também em outras condições, como por exemplo a coagulação intravascular disseminada.

A arteriografia pulmonar convencional (injeção de contraste radiológico na vasculatura pulmonar) embora seja considerada o padrão para o diagnóstico da embolia pulmonar é invasivo e pode provocar reações alérgicas e dano renal relacionados ao contraste radiológico. 


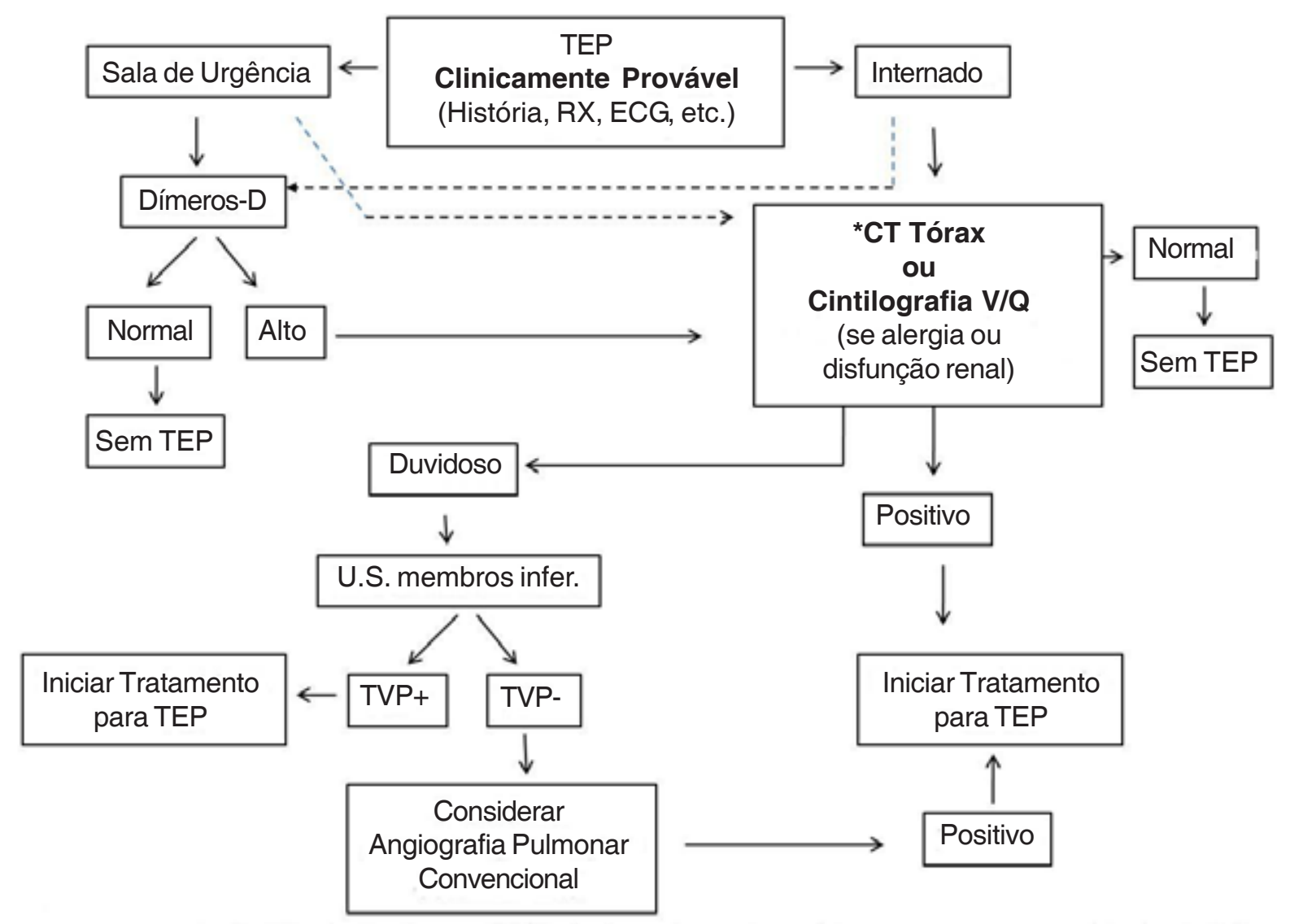

*Em pacientes instáveis e/ou impossibilidade de realizar CT, considerar ecocardiograma à beira do leito.

Figura 2. Algoritmo para o diagnóstico do tromboembolismo pulmonar. $\mathrm{TVP}=$ Trombose venosa profunda, TEP=tromboembolismo pulmonar

A cintilografia pulmonar de ventilação e perfusão, embora menos acessível, não é invasiva. Habitualmente este exame fornece um resultado que é relatado como "normal", "provável" ou "altamente provável" para o diagnóstico de embolia pulmonar a partir da análise de suas imagens. De forma simplista, na embolia as áreas acometidas apresentam captação da irradiação do radiofármaco nos alvéolos (ventilação presente), mas não há captação daquele no sistema arterial (perfusão ausente). No caso de uma atelectasia, por exemplo, teríamos captação do radiofármaco na vasculatura, mas não nos alvéolos.

Atualmente a angiografia obtida mediante tomografia computadorizada ("angioCT") de tórax vem ganhando espaço no diagnóstico do tromboembolismo pulmonar, sobretudo em decorrência da maior disponibilidade de aparelhos com de alta resolução e que possibilitam a rápida obtenção de imagens (tomogra- fia "Multi Slice"). A sensibilidade da angiotomografia pulmonar isolada é cerca de $83 \%$, mas quando associada a venografia computadorizada chega a $90 \%$.

Outro exame que pode se revelar muito útil, sobretudo em pacientes hemodinamicamente instáveis e/ou na impossibilidade da realização de tomografia computadorizada, é o ecocardiograma. O ecocardiograma, que poder ser realizado a beira do leito, pode fornecer sinais diretos e indiretos de tromboembolismo pulmonar, tais como sinais de sobrecarga e/ou disfunção ventricular direita ou mesmo a visualização de trombos na artéria pulmonar que, na vigência de sinais e sintomas clínicos sugestivos, podem autorizar o início de terapêutica específica.

\section{2- Profilaxia e Tratamento}

Basicamente a profilaxia do tromboembolismo pulmonar consiste na profilaxia da trombose venosa 
profunda, que deve considerar além do procedimento o risco do paciente. ${ }^{13} \mathrm{~A}$ abordagem detalhada da terapêutica foge ao propósito deste capítulo. Basicamente o tratamento é realizado com anticoagulação sistêmica parenteral na fase hospitalar seguida de anticoagu- lação crônica por via oral (warfarina) por 6 meses a um ano. Dependendo da gravidade pode-se lançar mão da utilização de trombolíticos associados à anticoagulação na fase hospitalar precoce, ou mesmo da tromboembolectomia cirúrgica, nos casos graves. ${ }^{11,14}$

Rodrigues AJ, Évora PRB, Vicente WVA. Postoperative Respiratory Complications. Medicina (Ribeirão Preto) 2008; 41 (4): 469-76.

ABSTRACT: Complications related to respiratory system are not uncommon after major surgeries and increase morbidity and mortality. Among the most frequent are atelectasis, pneumonia, respiratory failure and pulmonary thromboembolism. In this article we revised in brief the general aspects of pathophysiology, clinical presentation, diagnosis and clinical management of these conditions.

Key words: Postoperative Complications. Thromboembolism. Atelectasis. Pneumonia. Respiratory Insufficiency.

\section{REFERÊNCIAS}

1 - Lawrence VA, Cornell JE, Smetana GW. Strategies to reduce postoperative pulmonary complications after noncardiothoracic surgery: systematic review for the American College of Physicians. Ann. intern. med. 2006; 144: 596-608.

2 - Smetana GW, Lawrence VA, Cornell JE. Preoperative pulmonary risk stratification for noncardiothoracic surgery: systematic review for the American College of Physicians. Ann. intern. med. 2006;144: 581-95.

3 - Dayton MT. Surgical Complications. In: Townsend CM, Beauchamp RD, Evers BM, Mattox KL, editors. Sabiston Textbook of Surgery. Philadelphia: Elsevier Saunders, 2004.

4 - Duggan M, Kavanagh BP. Pulmonary atelectasis: a pathogenic perioperative entity. Anesthesiology. 2005; 102: 83854.

5 - Altemeier WA, Sinclair SE. Hyperoxia in the intensive care unit: why more is not always better. Curr. opin. crit. care. 2007; 13: 73-8.

6 - Lenz A, Franklin GA, Cheadle WG. Systemic inflammation after trauma. Injury. 2007; 38:1336-45.

7 - Ware LB, Matthay MA. The acute respiratory distress syndrome. N. Engl. j. med. 2000; 342: 1334-49.
8 - Arozullah AM, Khuri SF, Henderson WG, Daley J. Development and validation of a multifactorial risk index for predicting postoperative pneumonia after major noncardiac surgery. Ann. intern. med. 2001; 135: 847-57.

9 - Paintal HS, Kuschner WG. Aspiration syndromes: 10 clinical pearls every physician should know. Int. j. clin. pract. 2007; 61: 846-52.

10 - Ware LB, Matthay MA. Clinical practice. Acute pulmonary edema. N. Engl. j. med. 2005; 353: 2788-96.

11 - Goldhaber SZ. Pulmonary embolism. Lancet. 2004; 363: 1295305.

12 - Piazza G, Goldhaber SZ. Acute pulmonary embolism: part I: epidemiology and diagnosis. Circulation. 2006; 114: 28-32.

13 - Zurawska U, Parasuraman S, Goldhaber SZ. Prevention of pulmonary embolism in general surgery patients. Circulation. 2007; 115: 302-7.

14 - Piazza G, Goldhaber SZ. Acute pulmonary embolism: part II: treatment and prophylaxis. Circulation. 2006; 114: 42-7.

Recebido para publicação em 20/08/2008

Aprovado para publicação em 23/10/2008 